\title{
Experimental Detection of the Optical Pendellösung Effect
}

\author{
Maria L. Calvo, ${ }^{1, *}$ P. Cheben, ${ }^{2}$ O. Martínez-Matos, ${ }^{1}$ F. del Monte, ${ }^{3}$ and J. A. Rodrigo ${ }^{1}$ \\ ${ }^{1}$ Departamento de Óptica, Facultad de Ciencias Físicas, Universidad Complutense de Madrid, 28040 Madrid, Spain \\ ${ }^{2}$ Institute for Microstructural Sciences, National Research Council of Canada, K1A0R6 Ottawa, Canada \\ ${ }^{3}$ Instituto de Ciencia de Materiales de Madrid, Consejo Superior de Investigaciones Científicas (CSIC-UAM), \\ Canto Blanco, Madrid, Spain \\ (Received 11 April 2006; published 24 August 2006)
}

\begin{abstract}
We report observations of periodic oscillatory behavior of the angular selectivity, near the Bragg angle, in volume holographic gratings recorded in a new photopolymerizable glass with high refractive index modulation. We have detected the presence of overmodulation in the intensity distribution of the first diffraction order. The results reported here were achieved by incorporating in the photopolymerizable solgel glass zirconium-based high refractive index species at the molecular level. This is the first time that this effect is observed for light diffraction in an amorphous material.
\end{abstract}

DOI: 10.1103/PhysRevLett.97.084801

PACS numbers: $42.40 . \mathrm{Eq}, 42.40 . \mathrm{Pa}$

Introduction. - The Pendellösung effect was first observed by Shull in 1968 for the interference formed by the diffraction of slow neutron beams by thick perfect crystals of silicon with a thickness ranging from 0.33 to $1 \mathrm{~cm}$ [1]. The oscillatory behavior of the diffracted intensity was caused by the coherent transfer of neutron flux between the transmitted and internally Bragg-reflected beams by the periodic crystalline structure producing amplitude overmodulation. The Laue diffraction formalism provides the theoretical explanation of this phenomenon [2].

The Pendellösung fringe structure appears as a consequence of the interference between two branches of the wave function that result as solutions of the wave (Schrödinger) equation within the framework of the dynamical theory of diffraction. This phenomenon is formally analogous to the transfer of energy between two coupled pendulums, hence its name. One actual application of this effect was the experimental determination of the unit cell of the crystal structure and the scattering amplitude. The defects in the crystal structures (e.g., lattice dislocations) can hinder observation of the Pendellösung phenomenon since the presence of such defects can disturb the interference and the resulting energy transfer process. Similar experiments were later carried out on crystals other than silicon (e.g., Ge, $\mathrm{FeBO}_{3}$, and $\mathrm{Fe}_{2} \mathrm{O}_{3}$ ) and polarized neutron beams [3]. The Pendellösung effect has also been predicted and observed in second-order Bragg scattering of matter waves from a standing light wave acting as a thick grating [4]. Further experiments showed that a quasiPendellösung effect can be observed in the Raman-Nath regime for diffraction of atomic beams from standing light waves [5].

The dynamical theory of $\mathrm{x}$-ray diffraction formulated by Ewald [6] accounts for the Pendellösung effect. The coherent splitting of the incident beam will result in radiation periodically beating at different depths of the grating. The experimental observation of this effect is not trivial since it requires thick almost perfect crystal structures, or amor- phous highly modulated optical media, with large diffraction efficiency and dynamic range.

In 1993, we predicted that an optical Pendellösung effect should exist under certain restrictive conditions in a volume holographic grating, by considering a Gaussian and a plane wave interaction for the on-axis [7] and the off-axis and slightly off-Bragg regimes [8]. We showed that the beam splitting is due to the transverse gradient in the coupling coefficient describing the energy interchange between the diffracted and incident beams. A numerical estimation of the transverse intensity distribution at the output plane of the grating (similar to a lateral displacement in the scanning slit position) demonstrated that the oscillatory behavior is enhanced for gratings with a large thickness $(>50 \lambda)$ and high refractive index modulation $(\sim 0.01)$, assuming a small deviation from the Bragg angle. At that time, though, no amorphous holographic material was known with refractive index modulation and a thickness large enough to operate in a regime required for observation of the Pendellösung effect.

Overmodulation effects have been reported in unslanted holographic gratings recorded in silver halide emulsions (BB640 plates) and polyvinyl alcohol acrylamid photopolymers $[9,10]$. The authors explained the effect by the formation of a mixed (amplitude and phase) transmission holographic grating with correspondingly reduced diffraction efficiency. Recently, Mocella [11] has shown, via numerical simulation, that the refracted wave at the exit surface of a photonic crystal slab is periodically modulated, as a consequence of the Pendellösung effect.

In this Letter, we report for the first time, to the best of our knowledge, the observation of Pendellösung fringes in the optical domain in an amorphous photopolymerizable glass. The experimental results have been obtained with unslanted phase volume gratings holographically recorded in a new class of photopolymerizable glasses modified with high refractive index species (HRIS) [12]. We report on observation of two distinct characteristics of the optical 
Pendellösung effect. The first characteristic is the oscillatory behavior of the angular selectivity curve as a consequence of the index overmodulation. The oscillatory behavior is shown in Figs. 2(c) and 2(d) and is also compared to the angular selectivity response [dashed curve in Fig. 2(c)] in the absence of the overmodulation effect $(\Delta n=0.002)$. The second characteristic is the spatial modulation in the intensity distribution for light diffracted by an overmodulated sample, yielding a distinct fringe pattern, as is shown in Fig. 4.

Interestingly enough, an analog of this effect can be found in photorefractive media arising from nonlinear optically induced processes that give rise to the buildup of an internal space-charge field in the material, which, in turn, modifies the refractive index via the electro-optic effect [13]. Self-pumped phase conjugation was observed by Grunnet-Jepsen and co-workers in a photorefractive polymer with $140 \mu \mathrm{m}$ of thickness [14]. This capability for optical beams to interact inside a photomaterial and exchange both energy and phase information is fundamental in media exhibiting the photorefractive response. The transfer of energy from one beam to another during the recording process of a holographic grating (two-beam coupling) is regarded as an unambiguous test of photorefractivity [15]. In the soliton regime of beam propagation, a strong interaction among self-trapped light filaments has been predicted to occur in photorefractive silenites under fast alternating electric fields [16]. There, the development of light-induced abrupt refractive index changes was the key feature for strong beam energy exchange.

A new photopolymerizable glass modified with HRIS.In 2001, we reported a photopolymerizable sol-gel glass with a high diffraction efficiency and a large refractive index modulation $\Delta n$ and thickness [17]. Recently, we have reported an improved sol-gel composition with more than a twofold increase in the refractive index modulation. This remarkable improvement was achieved by incorporating the zirconium-based HRIS at the molecular level, resulting in a material with a very low scattering and a high optical quality while enhancing monomer diffusion [12]. High diffraction efficiency $(>90 \%)$ and high refractive index modulation $(\sim 0.01)$ have been obtained in samples of thicknesses up to $100 \mu \mathrm{m}$ with a negligible level of scattering. The hologram formation mechanism in this new material combines the conventional ColburnHaines-type monomer diffusion with a diffusion of $\mathrm{Zr}$ based high refractive index moieties, chelated with methacrylic acid, from the dark to the light regions of the interference pattern, hence producing a substantial increase in $\Delta n$, as required for the observation of the Pendellösung effect.

Recording of the volume holographic gratings. Figure 1 depicts the experimental setup for the recording of the holographic gratings. A standard interferometer setup with a Fresnel biprism was used. The grating was recorded with two $p$-polarized beams from a Nd:YAG laser of wavelength $\lambda=533 \mathrm{~nm}$. The grating was probed by a

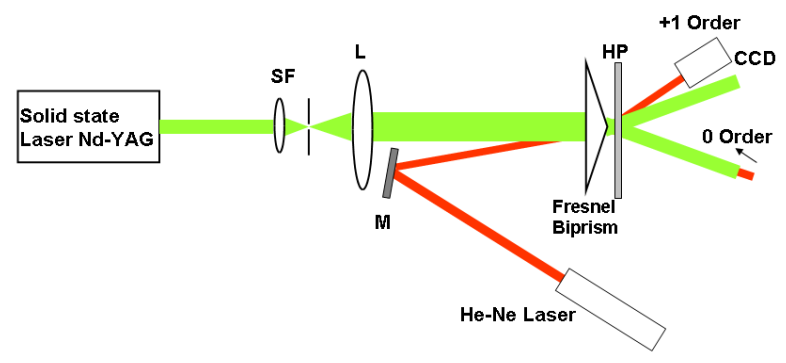

FIG. 1 (color online). Experimental setup for the recording of a holographic grating. Recording beam of $\lambda=533 \mathrm{~nm}$ [green (gray)] and readout beam of $\lambda=632 \mathrm{~nm}$ [red (black)]. SF: Spatial filter; L: lens; M: mirror; HP: holographic photomaterial; CCD: camera.

weak nonactinic $p$-polarized beam from a He-Ne laser of $\lambda=632.8 \mathrm{~nm}$. Gratings were recorded in several samples in order to analyze the influence of the thickness and refractive index modulation. After recording the grating, the dielectric permittivity of the holographic grating material can be expressed as:

$$
\varepsilon_{r}=\varepsilon_{r 0}+\varepsilon_{n}+\varepsilon_{r 1} \cos (\mathbf{K} \cdot \mathbf{r}),
$$

where $\varepsilon_{r 0}$ is the average dielectric permittivity of the material before exposure, $\varepsilon_{n}$ is the relative average increase after exposure, $\varepsilon_{r 1}$ is the dielectric permittivity modulation due to holographic recording, and $\mathbf{K}$ and $\mathbf{r}$ are the grating and position vectors, respectively. The energy flow in the grating region is given as the sum of two independent time averaged Poynting vectors associated to transmitted $S(x)$ and diffracted $R(x)$ waves. The diffraction efficiency for a transmission grating can be obtained from Kogelnik's theory [18] to a first-order approximation as proportional to $\sin ^{2}(\gamma)$, with $\gamma=$ $\pi \varepsilon_{r 1} d /\left(2 \lambda \varepsilon_{r 0}\right) \cos \theta_{h}$. Here $\theta_{h}$ is the Bragg angle, $d$ is the grating thickness, and $\lambda$ is the wavelength. However, this first-order approximation cannot account for the Pendellösung effect since the higher-order terms are neglected. The latter is accounted for by the dynamic diffraction theory that predicts a coupling term with an oscillatory behavior for nonabsorbing gratings, with an oscillation period determined by $\gamma$.

Experimental results. - Figure 2 shows the experimental results obtained in our photopolymerizable sol-gel glass incorporating the Zr-based HRIS. The recorded transmission unslanted gratings had a spatial period of 500 lines $/ \mathrm{mm}$. The Klein-Cook parameter [19] $Q \gg 10$ ( $Q=62$ in sample $1, Q=48$ in sample 2); hence, the Bragg regime holds. The gratings were probed using a HeNe laser with a spot size of approximately $15 \%$ of the grating size. The samples were illuminated in the center position of the grating.

As is shown in Figs. 2(a) and 2(b), the diffraction efficiency increases for the exposure range of $0-2.5 \mathrm{~J} / \mathrm{cm}^{2}$ [Fig. 2(a)] and $0-0.8 \mathrm{~J} / \mathrm{cm}^{2}$ [Fig. 2(b)], reaching a maximum value of $96 \%$. For the exposures above $2.5 \mathrm{~J} / \mathrm{cm}^{2}$ [Fig. 2(a)] and $0.8 \mathrm{~J} / \mathrm{cm}^{2}$ [Fig. 2(b)], the diffraction effi- 

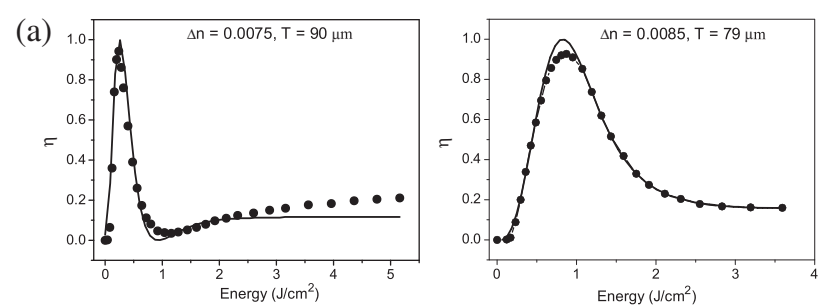

(b)
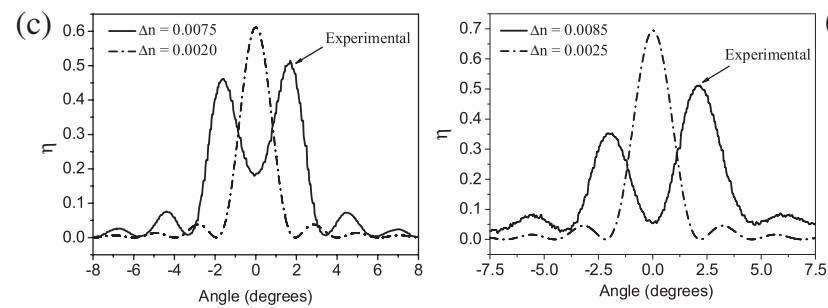

(d)

FIG. 2. Experimental evidence of diffracted light overmodulation in thick holographic gratings recorded in a photopolymerizable glass with large $\Delta n$. (a),(b) Diffraction efficiency evolution with exposure. Experimental results (data points) and coupled-mode theory fit (solid curves). (c),(d) Oscillation of the angular selectivity with the angle of incidence of the reading beam. $T$ : sample thickness, $\Delta n$ : refractive index modulation. All curves were obtained for the readout beam incident at the center of the grating.

ciency decreases as a consequence of the refractive index overmodulation. Notice the splitting of the peak of the angular selectivity response at the Bragg incidence and energy transfer to angular directions other than the Bragg angle yielding pronounced sidelobes in the angular selectivity curve, as observed in Figs. 2(c) and 2(d). These effects are distinct signatures of the overmodulation regime. Diffraction efficiency values of the angular selectivity for single maxima remained almost similar (theoretically, these values should coincide), in accordance with previous calculations [8]. In all measurements, the angle accuracy was $0.001^{\circ}$. The shapes of the angular distribution curves depend on the refractive index modulation and the thickness of the sample. The higher the modulation (for a given thickness), the higher the frequency of Pendellösung oscillations. The lack of symmetry is a result of the refractive index modulation gradient, i.e., varying $\Delta n$ with distance from the center of the hologram, as is shown in Fig. 3. As the result, diffraction efficiency slightly varies when the sample is rotated during the angular selectivity measurement. With an increasing overmodulation, the energy distribution in different propagation directions is modified, as is observed in Figs. 2(c) and 2(d). Notice that the energy transfer is maximized for directions corresponding to the sidelobes of the angular selectivity curve, i.e., directions other than the Bragg angle. The gratings were recorded with a slight refractive index modulation gradient along the transversal direction (see Fig. 3). Notice that the thickness gradient may vary as well. The values for $\Delta n$ and $T$ were obtained from Kogelnik's theory [18]. The gradient in $\Delta n$ does not noticeably affect the diffraction efficiency of the

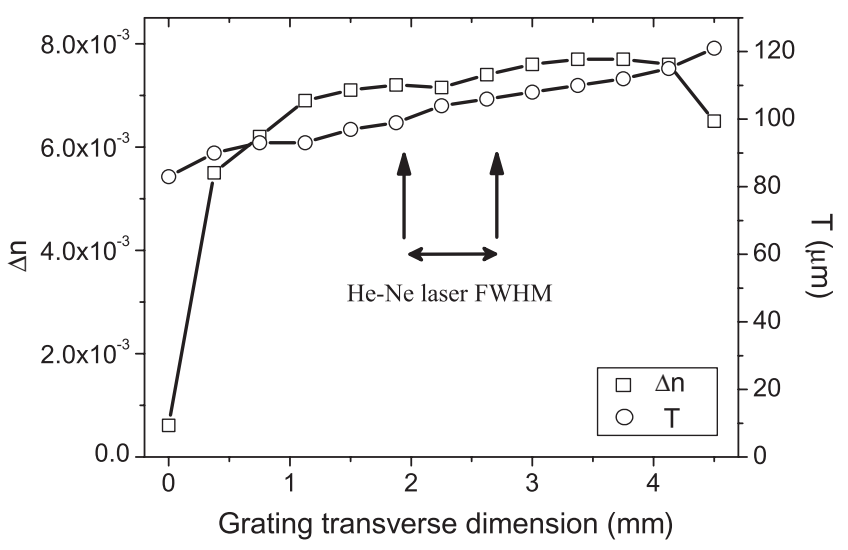

FIG. 3. Refractive index modulation $\Delta n$ (squares) as a function of the transverse dimensions of the grating in sample 1. Circles reproduce the slight variation of the sample thickness $T$. Results presented in Fig. 2 correspond to the illumination beam size region as indicated by the two arrows at FWHM.

readout beam when its size is small enough $(0.8 \mathrm{~mm}$ diameter at FWHM). Upon plane wave illumination of the grating, an index modulation gradient on the transverse distribution of the intensity of the diffracted beam was observed.

Figure 4 displays a sequence of recorded images at the position of the first diffracted order. Each image corresponds to the readout for an angular range $-10^{\circ}$ to $+10^{\circ}$ at steps of $1^{\circ}$ [as in Fig. 2(c)]. The observed fringes arise from the optical Pendellösung effect: A change of the incident beam angle in the presence of overmodulation will result in the energy transfer and, hence, in a change of the intensity modulation in the diffracted light pattern. The quality of the images is affected by the speckle background.

Conclusions.-We experimentally observed the Pendellösung effect for the diffraction of light by volume holographic gratings with high refractive index modulation and a high thickness, as previously predicted theoretically by us $[7,8]$. This has been achieved in a new class of photopolymerizable glass incorporating high refractive index moieties. The Zr-based HRIS allows for tailoring the material holographic properties to reach the overmodulation regime while maintaining a high diffraction efficiency. This observation of the Pendellösung effect demonstrates that the energy transfer, previously observed with neutron beams, $\mathrm{x}$ rays, and atomic beams, also exists in the optical domain (visible). Apart from their fundamental interest, our results suggest new routes for modal tailoring based upon strong refractive index modulation gradients. High efficiency mode converters for applications as diverse as microparticle manipulation with optical tweezers [20-22] to quantum information processing exploiting spatial modes carrying orbital angular momentum [23-25] seem feasible within our framework. For example, as is observed in Fig. 4, the light intensity spatial profile can be modified by tilting the sample (e.g., compare the fields for $\theta=-2^{\circ}$ 


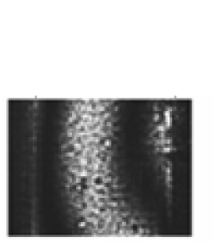

Grating Width
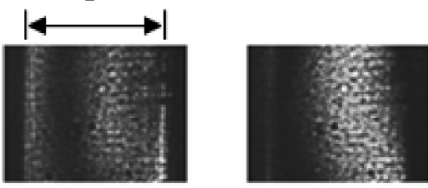

$\theta=-10^{\circ}$

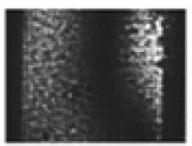

$\theta=-9^{\circ}$

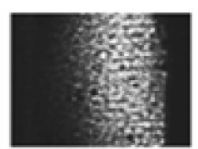

$\theta=-8^{\circ}$

$\theta=-7^{\circ}$

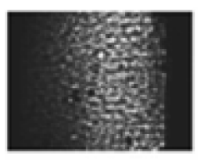

$\theta=-6^{\circ}$
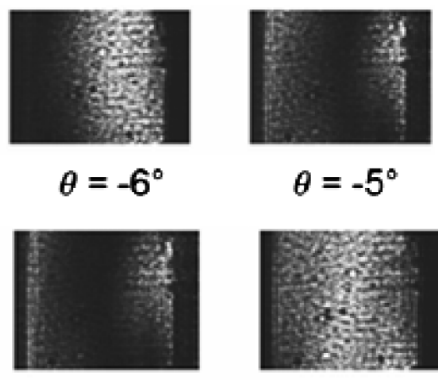

$\theta=-5^{\circ}$

$\theta=-4^{\circ}$
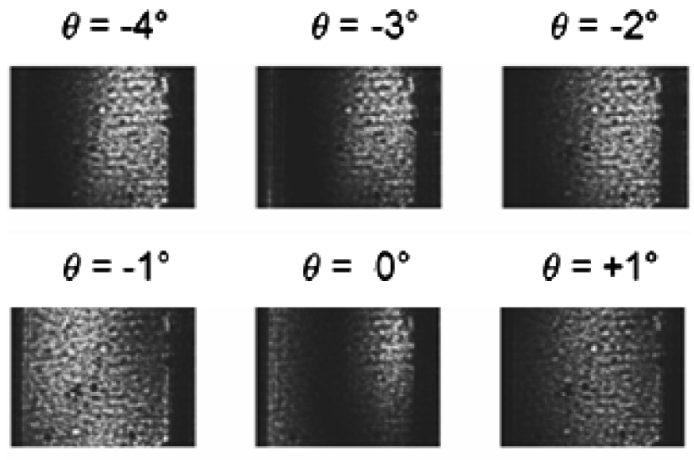

$$
\theta=+2^{\circ}
$$
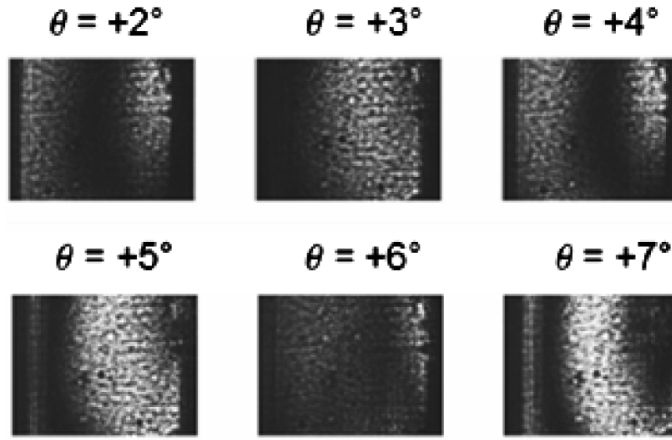

$\theta=+8^{\circ}$

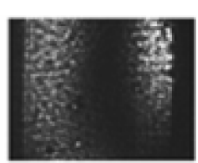

$\theta=+7^{\circ}$

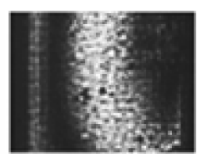

$\theta=+10^{\circ}$

FIG. 4. CCD images recorded at various angular positions of the grating. They correspond to the first diffracted order intensity distribution as reproduced while probing the grating with a plane wave and by varying the probe beam angle. The sequence covers the whole angular range as in Figs. 2(a)-2(c). The peaks of maximum energy correspond to $-2^{\circ}$ and $+2^{\circ}$, respectively. Dotted lines indicate the actual grating size $[5.3 \mathrm{~mm}$ width $=$ $12 \times 0.375 \mathrm{~mm}$ interval $+0.8 \mathrm{~mm}$ (see Fig. 3)].

and $-5^{\circ}$ ). This finding can be generalized since one may create a distinct two-dimensional intensity profile by multiplexing of different holograms, whereas the intensity profile for each hologram can be altered by the Pendellösung effect as shown in Fig. 4. The hologram multiplexing is allowed by the high dynamic range (refrac-

tive index modulation) available in our glass. We are pursuing studies to further clarify different aspects of this effect.

We thank G.F. Calvo for helpful discussions. Partial financial support from the Spanish Ministry of Education and Science through Projects No. TEC2005-02180 and No. MAT2003-02718 is gratefully acknowledged.

*Electronic address: mlcalvo@ fis.ucm.es

[1] C. G. Shull, Phys. Rev. Lett. 21, 1585 (1968).

[2] B. W. Batterman and H. Cole, Rev. Mod. Phys. 36, 681 (1964).

[3] V.F. Sears, Neutron Optics (Oxford University, New York, 1989), Sec. 6.3.

[4] S. Dürr, S. Kunze, and G. Rempe, Quantum Semiclass. Opt. 8, 531 (1996).

[5] C. Keller, J. Schmiedmayer, A. Zeilinger, T. Nonn, S. Dürr, and G. Rempe, Appl. Phys. B 69, 303 (1999).

[6] P. P. Ewald, Ann. Phys. (Berlin) 49, 1 (1916).

[7] P. Cheben and M. L. Calvo, J. Opt. Soc. Am. A 10, 2573 (1993).

[8] P. Cheben and M. L. Calvo, J. Opt. Soc. Am. A 13, 131 (1996).

[9] C. Neipp, I. Pascual, and A. Beléndez, J. Opt. A Pure Appl. Opt. 3, 504 (2001).

[10] C. Neipp, I. Pascual, and A. Beléndez, Opt. Express 10, 1374 (2002).

[11] V. Mocella, Opt. Express 13, 1361 (2005).

[12] F. del Monte, O. Martínez-Matos, J. A. Rodrigo, M. L. Calvo, and P. Cheben, Adv. Mater. 18, 2014 (2006).

[13] L. Solymar, D. J. Webb, and A. Grunnet-Jepsen, The Physics and Applications of Photorefractive Materials (Clarendon, Oxford, 1996).

[14] A. Grunnet-Jepsen, C. L. Thompson, and W. E. Moerner, Science 277, 549 (1997).

[15] W.E. Moerner, A. Grunnet-Jepsen, and C. L. Thompson, Annu. Rev. Mater. Sci. 27, 585 (1997).

[16] G.F. Calvo, B. Sturman, F. Agulló-López, and M. Carrascosa, Phys. Rev. Lett. 89, 033902 (2002).

[17] P. Cheben and M.L. Calvo, Appl. Phys. Lett. 78, 1490 (2001).

[18] H. Kogelnik, Bell Syst. Tech. J. 48, 2909 (1969).

[19] W. R. Klein and B. D. Cook, IEEE Trans. Sonics Ultrason. 14, 123 (1967).

[20] H. He, M.E.J. Friese, N.R. Heckenberg, and H. Rubinsztein-Dunlop, Phys. Rev. Lett. 75, 826 (1995).

[21] V. Garcés-Chávez, D. McGloin, M. J. Padgett, W. Dultz, H. Schmitzer, and K. Dholakia, Phys. Rev. Lett. 91, 093602 (2003).

[22] D. G. Grier, Nature (London) 424, 810 (2003).

[23] A. Mair, A. Vaziri, G. Weihs, and A. Zeilinger, Nature (London) 412, 313 (2001).

[24] A. Vaziri, J.-W. Pan, T. Jennewein, G. Weihs, and A. Zeilinger, Phys. Rev. Lett. 91, 227902 (2003).

[25] N. K. Langford, R. B. Dalton, M. D. Harvey, J. L. O’Brien, G. J. Pryde, A. Gilchrist, S. D. Bartlett, and A. White, Phys. Rev. Lett. 93, 053601 (2004). 